Lack, C. H. \& Tanner, F. (1953). J. gen. Microbiol. 8, 18-26.

\title{
The Significance of Pleomorphism in Mycobacterium tuberculosis var. hominis
}

\author{
By C. H. LACK AND F. TANNER \\ The Institute of Orthopaedics, Royal National Orthopaedic \\ Hospital, Stanmore, Middlesex
}

SUMMARY: Under adverse conditions, Mycobacterium tuberculosis may autolyse, or surviving cells may divide into progressively shorter cells which may even appear to be coccal. We have not observed a complex 'life cycle', but the cells whose cytoplasm has been reduced show qualitative differences which we term 'regression'.

The significance of non-acid-fast forms, granules, beads, 'substance cyanophil' and spore-like bodies, observed in cultures of Mycobacterium tuberculosis, and in tubercular material, has been one of the major controversies of medical bacteriology during the past fifty years.

Our interest in this problem arose from frequent observation of acid-fast coccal forms in pus from tuberculous lesions of bone in which no bacilli could be found. We decided to investigate the morphological variability of human strains of $\boldsymbol{M}$. tuberculosis under different conditions, and to see whether we could induce changes corresponding to any of the 'life cycles' previously described. Some of the conditions which we imposed on our cultures were chosen because they were said to be successful in inducing the $\mathrm{L}$ cycle of other bacteria.

\section{MATERIALS AND ME'THODS}

Phase-contrast microscopy. A Beck microscope with a $2 \mathrm{~mm}$. objective and $\times 10$ eyepiece, and illuminated by a $6 \mathrm{~V}$. Tenslite with an Ilford 404 green filter was used for intermittent observations, and two Cooke microscopes for the observation of microcultures over longer periods. The larger of these was described in this Journal (Lack, 1952). The other has a Newman-Waterfield warm chamber (Kaylene Ltd. London) which maintains a slide culture at $37^{\circ}$. Both these microscopes are fitted with Cooke phase-contrast systems and Ilford green filters.

Staining techniques. Ziehl-Neelsen, with and without counterstain, Much's and Gram's methods and $1 \%$ malachite green counterstained by $\mathbf{0 . 5} \%$ safranin. Klieneberger-Nobel's (1950) techniques of agar cut-out and osmic acid vapour fixation were used for cytological studies.

Microcultures. The majority were made on Dubos agar prepared by the addition of $1.5 \mathrm{~g}$. New Zealand agar to $90 \mathrm{ml}$. Tween-Dubos medium, which was steamed for $1 \mathrm{hr}$., adjusted to $\mathrm{pH} 7 \cdot 2$, filtered, and autoclaved at $10 \mathrm{lb}$. pressure for $10 \mathrm{~min}$. Bovine albumen solution was added at $55^{\circ}$ to give a final concentration of $1 / 26$.

Strains. H37 Rv was used in most of this work, but a large number of strains has been freshly isolated from patients. 
Culture media. Löwenstein, Dubos, and Bordet Gengou with $50 \%$ human red cells, were used most frequently. In order that pleomorphism might be studied under various controlled conditions, the growth of $\mathrm{H} 37 \mathrm{Rv}$ was also observed in the following media: Dubos, Dubos agar, glucose peptone water, glucose broth, $10 \%$ glycerol in Dubos, yeast agar, $1 \%$ urea in Dubos, $1.5 \%$ glycine in Dubos. Cultures were made in urea-Dubos because granular forms were frequently observed in urine. Glycine was tried because it has been found to favour the production of L-forms in some other genera.

Strain H37 Rv was also grown in the following biological fluids : serum from tuberculous and non-tuberculous patients; serum inactivated at $56^{\circ}$ for $30 \mathrm{~min}$.; pus digested with plasmin and deoxyribonuclease; synovial, pleural and amniotic fluids. The action of physical and chemical agents and of various antibiotics was also studied.

\section{OBSERVATIONS}

In eighty-eight experiments the growth of different strains was observed in a wide range of media. Only in media containing glycine or antibiotics did we observe the development of giant forms, and under no conditions did we observe a true life cycle in any way resembling that of fungi. Although the variations in pellicle production in liquid media were striking, and merit more attention, they were not studied further since they were not connected with morphological variation.

The Tables set out a summary of some of our observations representing the changes observed (i) in exhausted or deficient media (Table 1); (ii) when grown with different antibiotics (Table 2). These changes were observed mainly by means of phase contrast, often over long periods; if the photographs (Pls. 1-3) are studied before the Tables, terms such as typical bacilli (Pl. 2, fig. 5), autolysed rafts (Pl. 1, figs. 1, 2), degenerate bacilli (PI. 1, fig. 3), short forms and 'diphtheroids' (Pl. 2, figs. 6a,b), and coccal forms (Pl. 1, fig. 4 and Pl. 2, fig. 7) will be better understood. Unless a stain is mentioned (z.N. = ZiehlNeelsen, M.G.s. = malachite green safranin), the description is that of the phase-contrast preparation.

Multiplication. We saw each of the methods of multiplication of bacilli described by $\mathrm{Hu}$ (1936), and illustrated diagrammatically (Fig. 1a). Lateral budding was rare. Under some adverse conditions, surviving cells continued to divide in these ways, but as they did not grow so long, the daughter cells produced the coccal groupings that have been so frequently described (Fig. $1 b$ ). In very old cultures or under the action of some antibiotics, cells sometimes grew so little before they divided that they were already cocciform before they sent out a bud; these Hu described as 'sprouting granules'.

Autolysis. We watched the changes which occur when cells autolyse, and correlated the appearances seen by phase contrast with corresponding stained preparations. When individual cells were about to lyse, their nuclei became spherical and distended the cell wall (Pl. 1, fig. 3), giving the appearance of spores. When, on the other hand, the cells were in a cord or raft, autolysis began in one portion while cells elsewhere were still growing and dividing. 
The outlines of the dead cells disappeared, the cytoplasms of adjacent cells fused and the nuclei became globular. The globules in this symplasm varied in size just as the nuclei varied in the cells before autolysis (PI. 1, fig. 1). As the symplasm shrank and the nuclear globules were drawn closer together the mass had the appearance of a cluster of cocci (Pl. 1, fig. 2). These products of autolysis could persist for a very long time. Often they were embedded in

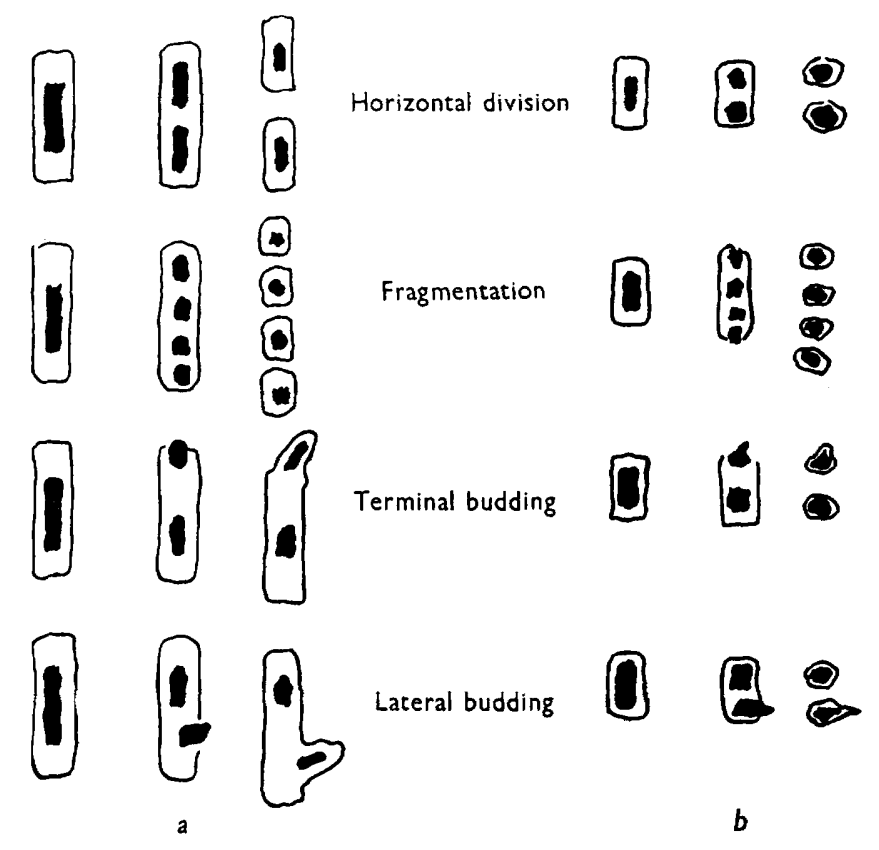

Fig. 1

long strands of extracellular material (slime, 'substance cyanophil'). If bacilli were still dividing close to an area of autolysis, they were likely, for reasons we give below, to be short or even coccal in form, so that it might appear as though they had their origin from this symplasm. We never observed regeneration from cells which had undergone autolysis, either individually or en masse.

Though subcultures and observation of growth in microcultures were used to check viability, we found during the course of these observations that the malachite green safranin stain was quite a reliable indication of the state of a culture-viable bacilli staining green and dead or dying bacilli staining pink, though both might have stained red with the orthodox Ziehl-Neelsen stain. In this connexion we confirmed Nedelkovitch's observation that uninoculated egg media may contain acid-fast material which, after staining with ZiehlNeelsen, can easily be mistaken for the smaller forms of $M$. tuberculosis (Nedelkovitch, 1950).

The biological fluids listed all appeared to retard growth with the exception of digested pus. Treatment of tuberculous pus with 'streptokinase-strepto- 


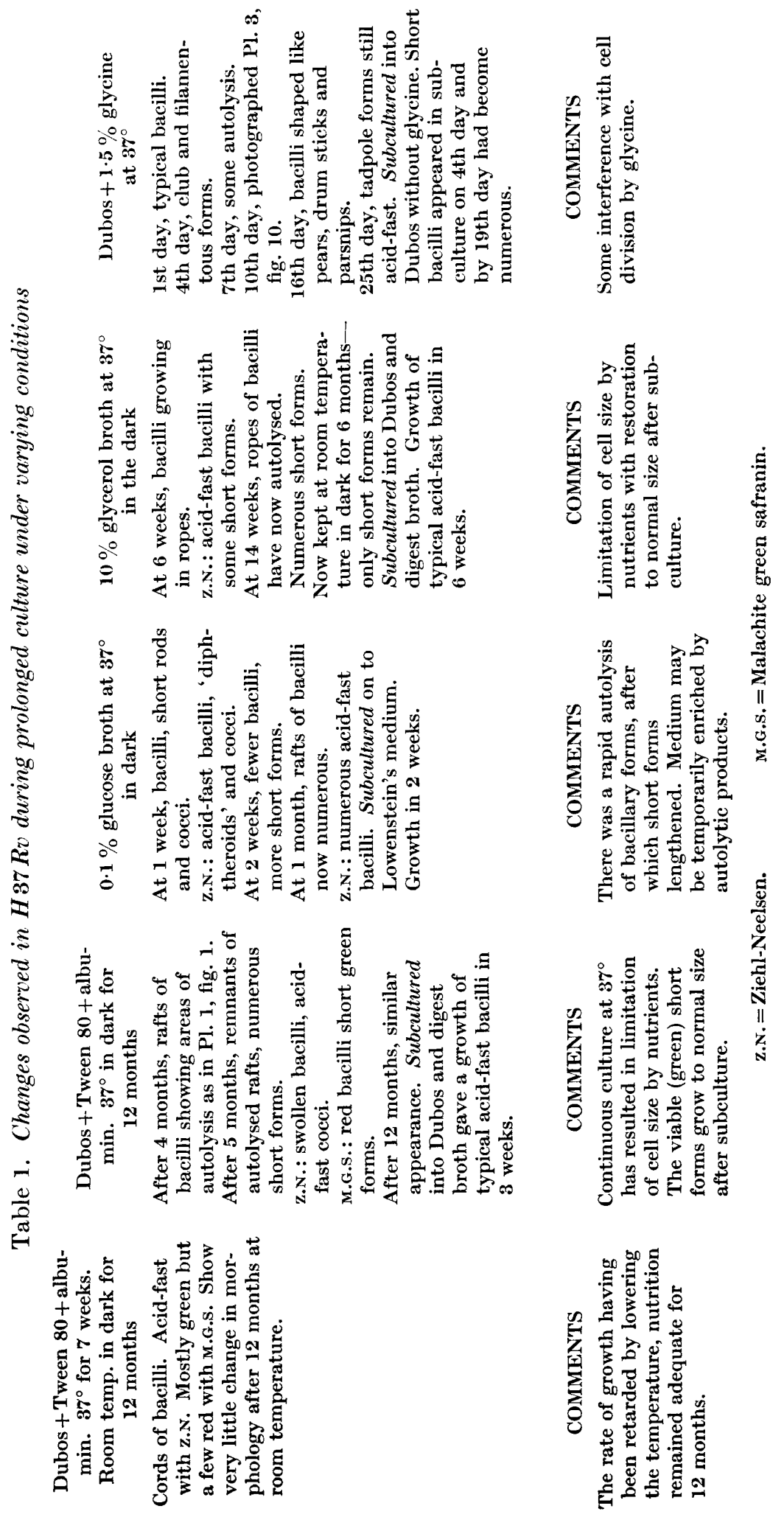



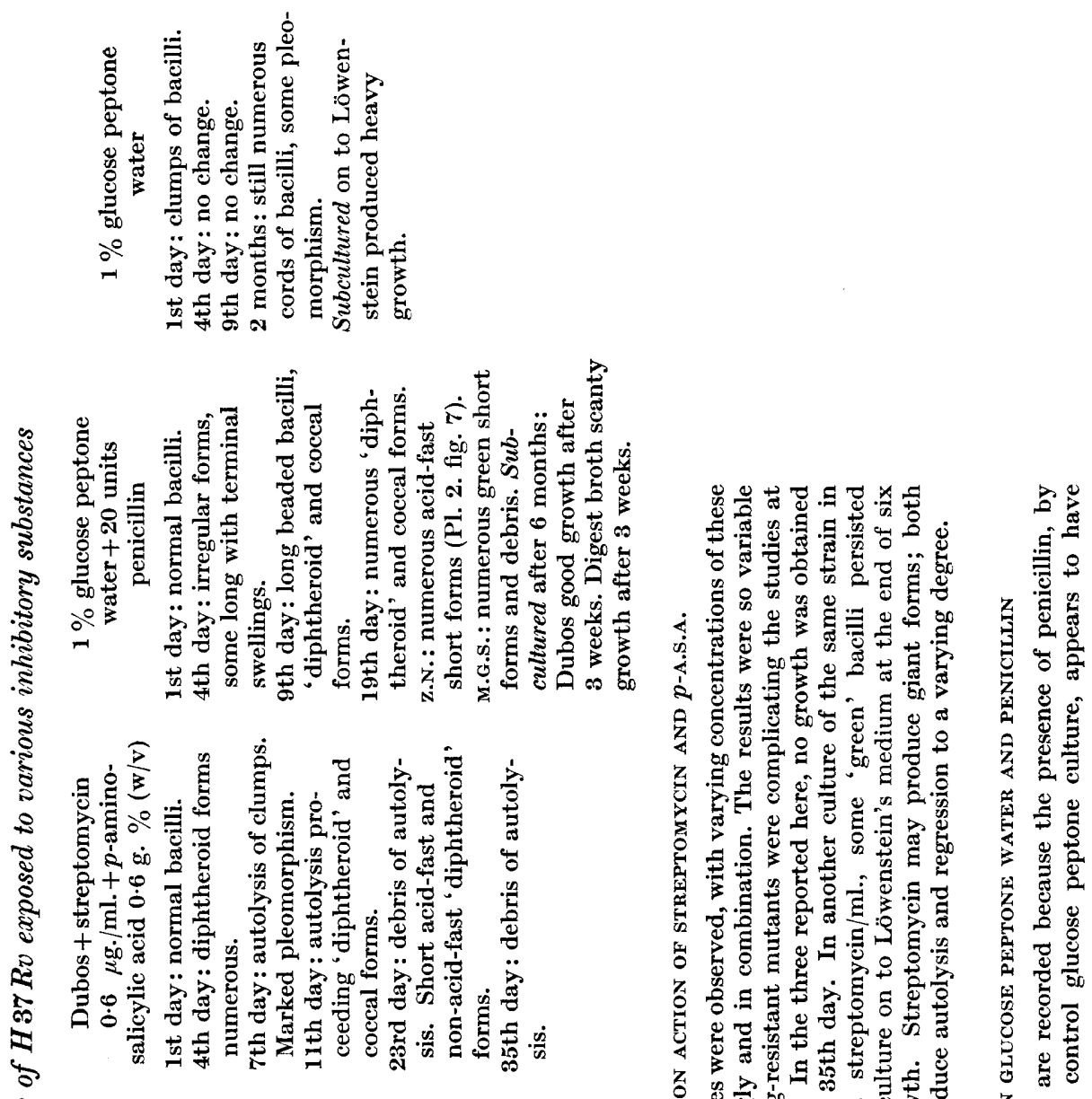

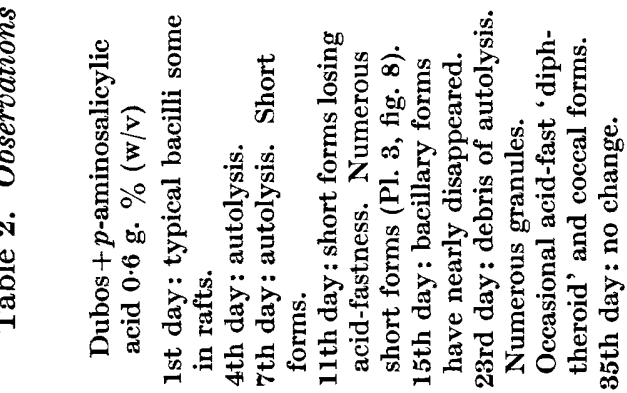
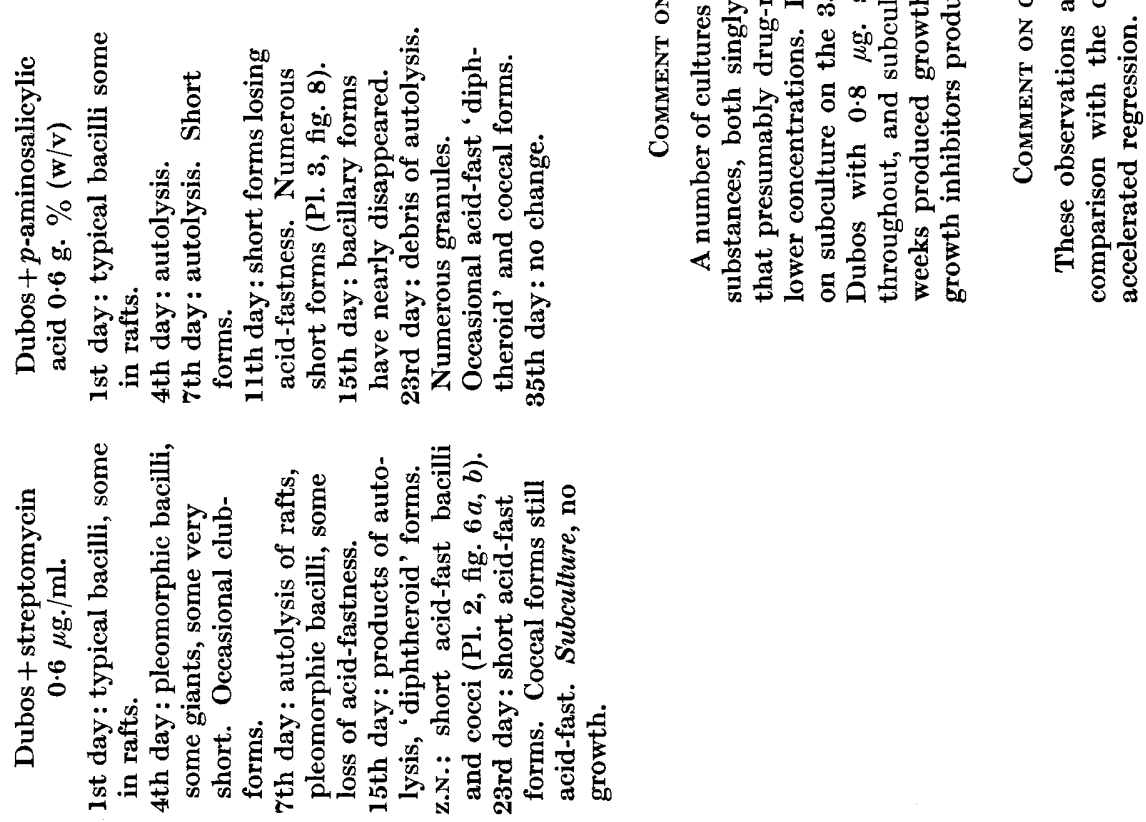
dornase' (Burroughs Wellcome and Co.) enhanced the growth of tubercle bacilli and permitted degraded forms to grow on Löwenstein medium that otherwise would not (Tanner, 1951).

We have begun the study of morphological changes that occur immediately after inoculation into animals. A fuller account of this will be reported later, but attention may be drawn to the photograph (Pl. 3, fig. 9) of the 'diphtheroid' type of bacilli recovered from a rabbit's peritoneal exudate 10 days after intraperitoneal inoculation of a suspension of living bacilli. The strain $\mathrm{H} 37 \mathrm{RV}$ was inoculated intraperitoneally into rabbits, guinea-pigs and rats, and their peritoneal exudates were examined at intervals. No fully developed acid-fast bacilli could be found in the deposits of these exudates in the early stages, but numerous short acid-fast rods and cocci, both free and in macrophages, were seen, and culture on Löwenstein gave a luxurious growth of tubercle bacilli.

Lastly, our findings are illustrated in one of the number of clinical infections which provoked this study. A young man noticed a painless swelling on his right knee following exercise. This was treated by diathermy for 2 months without benefit. After 3 months a biopsy of the synovial membrane of this joint was taken. Histological examination showed no inflammatory reaction and no evidence of tuberculosis. Staining by the Ziehl-Neelsen method showed cocco-diphtheroid forms in the synovial membrane and fluid (Pl. 3, fig. 11), and inoculation of this material into a guinea-pig produced tuberculosis with typical acid-fast bacilli in the guinea-pig spleen (Pl. 3, fig. 12).

\section{DISCUSSION}

The two conclusions that we have reached from these studies are $(a)$ that the process and appearance of autolysis has confused many workers in the past and discounts much of what has been written about stages in a 'life cycle', and $(b)$ that the progressive shortening of bacilli under some adverse conditions, a process which we propose to call 'regression' instead of the usual term 'degeneration', represents not merely a reduction in size of the organisms but qualitative changes that have important implications.

Grigoraki (1950) seems to have confused these two processes. In his account of the sexual phase of development, the ectoplasm of lysed bacilli fused to form an amorphous veil in which the nuclei were transformed into granules which fused to form a symplasm. He describes stalks growing out from the symplasm bearing large ovoid cells which on bursting release male and female cocciform gametes. Short rods are supposed to form by fusion of these gametes. It seems more likely that the fusion and lysis of cells and the release of the nuclei therefrom is a process of autolysis, and that the short rods are derived from bacilli which have escaped autolysis, though they may still be attached to, and appear to grow out from, a clump of bacilli which have lysed.

Pl. 1, figs. 1 and 2, illustrate how easily such a confusion may arise, particularly if the preparation had been fixed and stained instead of being observed by phase contrast. Pl. 1, fig. 1, shows the process of autolysis in a raft of bacilli. Autolysis is spreading from right to left in this picture but 
'coccal' and 'diphtheroid' forms derived from dividing bacilli are appearing in the top left-hand quadrant.

When the symplasm of an autolysing raft has disappeared and the nuclei are clumped together, as we see in Pl. 1, fig. 2, the multiplication of a few surviving bacilli may well appear to result from the fusion of 'coccal forms'.

Schaefer, Marshak \& Burkhart state that in the absence of antibiotics, exhaustion of the nitrogen source in the culture medium is an essential condition for the appearance of autolysis, and that both oxygen and a source of energy such as glucose are necessary (Schaefer, Marshak \& Burkhart, 1949; Marshak \& Schaefer, 1952). In the presence of streptomycin, penicillin and $p$-amino salicylic acid, autolysis always occurs; giant forms and monstrosities appear in the presence of streptomycin and of glycine.

The important issue in this discussion is the significance of the coccal forms. We wish to emphasize that there may be three kinds of 'coccal' forms in a stained preparation: $(a)$ nuclear remnants from autolysed bacilli appear 'coccal'; $(b)$ in stained preparations coccal forms may be produced by the detachment of growing tips of bacilli in the smearing of cultures (these may frequently be observed as round bodies close to the growing end of mature bacilli, and may be regarded as artefacts-Yegian \& Porter, 1944); $(c)$ the coccal forms which are of the greatest interest are those which are produced in large numbers under adverse conditions by the division of diphtheroid forms, and which stain green with the M.G.s. stain.

Bisset's (1949) view is that tubercle bacilli are multicellular, a single bacillus containing up to twelve or more cells. We agree that this is so when it is fragmenting, but in our studies of human strains this mode of multiplication is uncommon. Growing from the tip of a bacillus with subsequent snapping of the newly formed bacillus to a $\Lambda$ is seen most frequently among isolated bacilli, whereas in clumps the added bacilli form cords. Under adverse conditions, fragmentation, transverse division and lateral budding have all been observed, and as the bacilli become progressively shorter, multiplication in these ways gives rise to 'coccal' groupings.

A statement that cells diminish in size as they divide in an inadequate medium may appear so trite as to merit no further consideration. That the reduction in nitrogen content may be very considerable is shown by the findings of Marshak \& Schaefer (1952) who report that 'in the presence of adequate amounts of glucose in a medium deficient in a source of nitrogen, tubercle bacilli continue to proliferate', and that 'the cells of the second generation (fourth day) produced under these conditions have a nitrogen content per milligram of dry weight which is only approximately $40 \%$ of that in the cells used as inoculum. Relatively little of the cellular nitrogen originally added is lost to the medium.' The weight of the culture was nearly doubled and the final density was $2 \cdot 6$ times the initial density. They did not report their observations on the morphology of these organisms, and their statement as it stands might be taken to imply a $2 \frac{1}{2}$-fold relative increase in non-nitrogenous cell constituents. However, we find a marked reduction in size of dividing cells not only with nitrogen starvation but also in the presence 
of antibiotics and biological fluids, and from our few initial observations it appears that bacilli dividing inside macrophages also tend to become diphtheroid or coccal. These coccal forms are capable of growing into full-bodied bacilli when transferred to a suitable environment.

Vaudremer (1926) showed that tubercle bacilli, when grown on poor media, become non-acid-fast and pleomorphic and cease to produce tuberculin. On return to suitable media they recovered their acid-fastness. Pagel (1934) found in histological sections of tuberculous foci slender chromophilic bacilli in some lesions and short plump chromophobic bacilli or granules in others. The former produced more rapid development and larger tuberculous infiltrations in guinea-pigs than the latter. We also found a striking difference in the morphology of bacilli from tuberculous sinuses where the bacilli are large and well formed, and those from closed lesions in bone where the organisms are usually coccal. The former produced rapid development of tuberculosis in guinea-pigs, the latter slowly or not at all; since the use of streptomycin, organisms seen in tuberculosis pus from sinuses are now usually like those from bone, that is, short, poorly acid-fast and with low infectivity.

Brieger, Miles, Cosslett \& Horne (1951) recovered granular forms of bovine tubercle from the spleens and lungs of infected rabbits and found these to be infective to guinea-pigs, though they would not grow on laboratory media. Kahn \& Nonidez (1936) found that culture filtrates of H37 and of a bovine strain that had been through membrane filters of a pore volume from $\mathbf{0 . 5}$ to $0 \cdot 1 \mu$ caused tuberculosis in four out of five guinea-pigs, though inoculations on to Petroff's, Long's, Löwenstein's and glycerol agar medium were sterile. We have also encountered the anomaly of granular forms in infected material which would not grow on routine media but which infected guinea-pigs, though in this respect growth has occurred on Bordet Gengou's medium with $50 \%$ human red cells added, when the other media have remained sterile.

We suggest that tubercle bacilli, carried by the blood from a primary or secondary focus, may lodge in areas in the skeletal system and in other sites in which the micro-environment is unfavourable for full development, and that there the organisms may regress and remain dormant until the environment is altered in their favour by injury, haemorrhage, metabolic changes or infection by other organisms.

We have found short diphtheroid and coccal forms staining green with the malachite-green safranin in which no bacilli or tubercles could be found in joint tissue biopsies, and this tissue has infected guinea-pigs with tuberculosis. The patients have invariably been tuberculin-positive (evidence of an antecedent infection), but showed no signs of an active tuberculosis until after trauma. In our view, this suggests that the organisms were in the joint tissues in a form that did not produce a characteristic reaction even in sensitized hosts until the change in their environment allowed them to grow into bacilli again. Once this has occurred the characteristic tissue reaction follows. 


\section{REFERENCES}

Bisset, K. A. (1949). Observations upon the cytology of corynebacteria and mycobacteria. J. gen. Microbiol. 3, 93.

Brieger, E. M., Miles, J. A. R., Cosslett, V. E. \& Horne, R. W. (1951). Nature and infectivity of granular forms of the bovine tubercle bacillus. Nature, Lond.168, 96.

Grigoraki, L. (1950). Colloid Chemistry. New York: Reinhold Publ. Co. 313.

$\mathrm{Hu}, \mathrm{K}$. (1936). Study of the method of development of bacteria by means of a single cell culture in a glycerine-agar film. Jap. J. exp. Med. 14, 29.

KAHN, M. C., \& NonidEz, J. F. (1936). The role of non-acid fast rods and granules in the developmental cycle of the tubercle bacillus. Amer. Rev. Tuberc. 29, 389.

KLIENeberger-Nobel, E. (1950). Methods for the study of the cytology of bacteria and pleuropneumonia-like organisms. Quart. J. micr. Sci. 91, 340.

LACK, C. H. (1952). A simple hot box for microcultures. J. gen. Microbiol. 7, 144.

MARShak, A. \& Schaefer, W. B. (1952). Comparison of growth of autolysis of two strains of tubercle bacilli. Amer. Rev. Tuberc. 65, 75.

NedElkovitch, J. (1950). Method of multiplication of Koch's bacillus. Ann. Inst. Pasteur, 78, 177.

PAGeL, W. (1934). Histological studies on the variability of the tubercle bacillus. J. Path. Bact. 39, 689.

Schaefer, W. B., Marshak, A. \& Burkhart, B. (1949). The growth of Mycobacterium tuberculosis as a function of its nutrients. J. Bact. 58, 549.

Tanner, F. (1951). A quick and safe method for culturing Myco. tuberculosis from pus. J. med. Lab. Tech. 9, 226.

VAUDREMER, A. (1926). Le développement du bacille tuberculeux et ses conséquentes. Rev. Med., Paris, 43, 419.

Yegian, D. \& Porter, K. R. (1944). Some artifacts encountered in stained preparations of tubercle bacilli. I. Non-acid fast forms arising from mechanical treatment. J. Bact. 48, 83.

\section{EXPLANATION OF PLATES}

\section{Plate 1}

Fig. 1. Autolysis within a raft of bacilli. H37Rv. 3-week Dubos culture. Phase contrast. $\times 4000$.

Fig. 2. Autolysis at a later stage: the nuclei resemble a cluster of cocci. H37 Rv in Dubos with streptomycin and $p$-A.S.A. Phase contrast. $\times 4000$.

Fig. 3. H37 Rv in Dubos. 2 months. Bacilli about to lyse.

Fig. 4. H37 Rv. 1 year in glucose peptone water-still viable. Short forms resembling cocci. Much's stain. $\times 2000$.

\section{Plate 2}

Fig. 5. H37 Rv. 14-day Dubos culture-normal bacilli. z.N. stain. $\times 2000$.

Fig. $6 a, b$. H37 Rv. 15-day culture in Dubos plus 0.17 $\mu$ g. streptomycin/ml. Short forms predominate. z.N. stain. $\times 2000$.

Fig. 7. H37 Rv. 19-day culture in glucose peptone water plus 20 units penicillin $/ \mathrm{ml}$. Short forms and autolysis. z.N. stain. $\times \mathbf{2 0 0 0}$.

\section{Plate 3}

Fig. 8. Short forms of H37 Rv grown in Dubos with $p$-A.S.A. Phase contrast. $\times 4000$.

Fig. 9. Short forms of $\mathrm{H} 37 \mathrm{Rv}$ recovered from rabbit peritoneal exudate 10 days after intraperitoneal inoculation. Phase contrast. $\times 4000$.

Fig. 10. H37 Rv in Dubos plus $1.5 \%$ glycine for 10 days. Abnormal nuclei. $z . N . \quad \times 2000$.

Fig. 11. Acid-fast cocco-diphtheroid forms in the synovial fluid of a patient in which no acid-fast bacilli were found. z.N. stain. $\times 3000$. Ilford micro 2 filter has been used to reduce the density of the surrounding cells.

Fig. 12. Acid-fast bacilli in the spleen of a guinea pig injected with the fluid illustrated in Fig. 11. Z.N. stain. $\times 3000$.

(Received 3 March 1952) 
Journal of General Microbiology, Vol. 8, No. 1
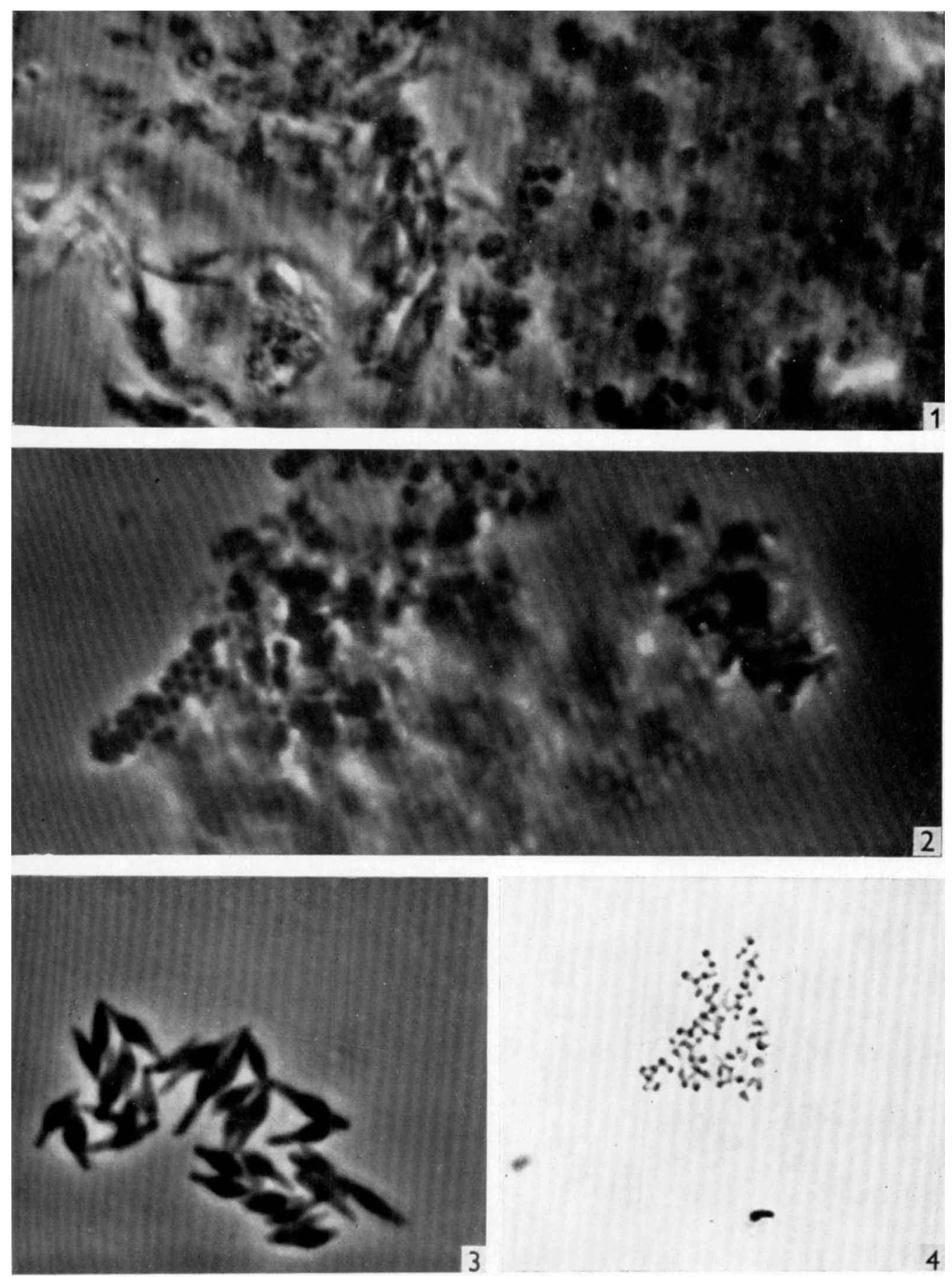

C. H. Lack \& F. Tanner-Pleomorphism of M. teberculosis. Plate 1 
Journal of General Microbiology, Vol. 8, No. 1

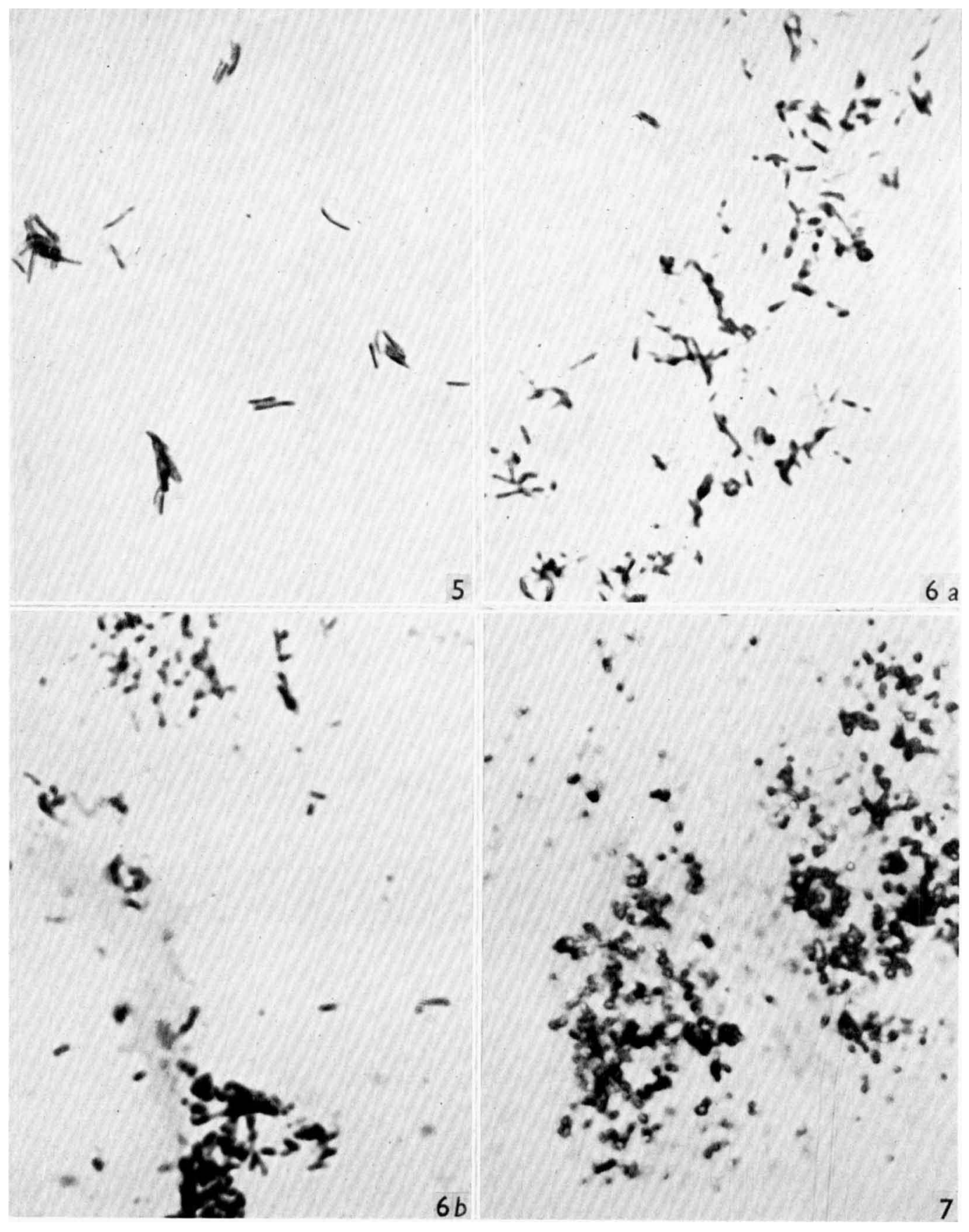

C. H. Lack \& F. 'Tanner-Pleomorphism of $M$. tuberculogis. Plate 2 
Journal of General Microbiology, Vol. 8, No. 1
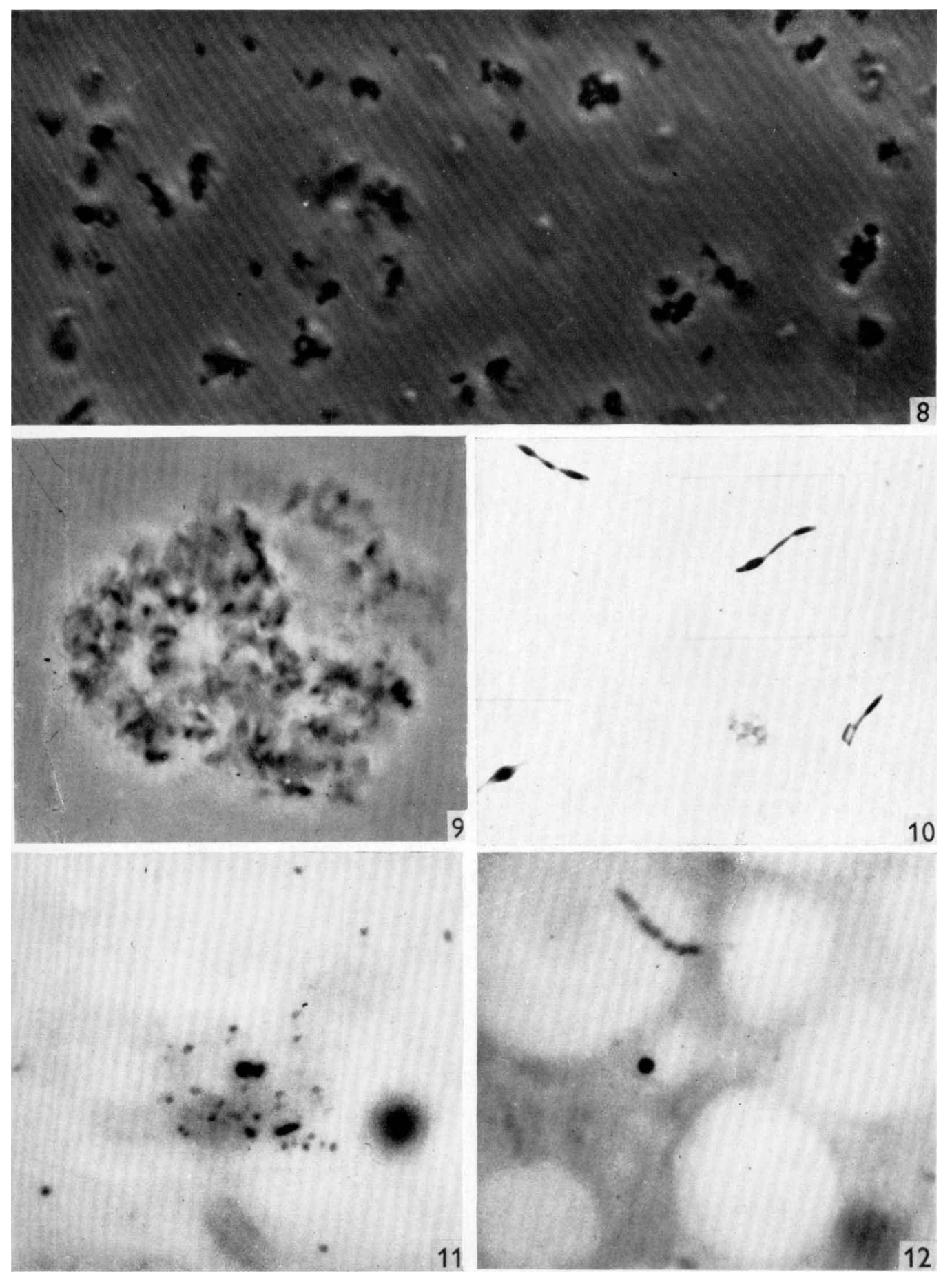

C. H. Lack \& H. 'Tanver-Pleomorpingm of M. Thberclosts. Piate: 